\title{
Inelastic neutron scattering spectroscopy of amino acids
}

\author{
Stewart F. Parker ${ }^{\mathrm{a}, *}$ and Parvez I. Haris ${ }^{\mathrm{b}}$ \\ a ISIS Facility, STFC Rutherford Appleton Laboratory, Chilton, Didcot, Oxon OX11 0QX, UK \\ ${ }^{\mathrm{b}}$ Faculty of Health \& Life Sciences, De Montfort University, Leicester, LE1 9BH, UK
}

\begin{abstract}
A combination of infrared, Raman and inelastic neutron scattering (INS) spectroscopies are used to provide complete vibrational spectra of several amino acids and dipeptides. The amino acids studied were glycine, alanine, glutamine, cysteine, methionine and phenylalanine and the dipeptides studied were Gly-Gln and Gly-Ala. The findings of this study have shown how the complementarity of infrared, Raman and INS spectroscopies can be exploited to provide complete vibrational spectra of amino acids and peptides. In particular, the strengths of INS spectroscopy are highlighted: the absence of selection rules, that hydrogenic motions are emphasised, the ready access to the low energy regime $\left(<400 \mathrm{~cm}^{-1}\right)$ and the straightforward calculation of intensities. In the future, it should be possible to apply this approach to the study of larger peptides as well as proteins.
\end{abstract}

Keywords: Infrared, Raman, inelastic neutron scattering spectroscopy, amino acids, peptides, proteins, density functional theory

\section{Abbreviations}

DFT, density functional theory;

INS, inelastic neutron scattering.

\section{Introduction}

Infrared and Raman spectroscopies are frequently used for the investigation of biological systems [1] because they provide detailed information about the local structure of the material. However, most of the focus is on a few key bands, usually the amide $\mathrm{I}$, a coupled $\mathrm{C}=\mathrm{O}$ stretch and $\mathrm{N}-\mathrm{H}$ bend and the amide III, a coupled $\mathrm{C}-\mathrm{N}$ stretch and $\mathrm{C}-\mathrm{N}-\mathrm{H}$ in-plane bend and, to a lesser extent, the $\mathrm{C}-\mathrm{H} / \mathrm{N}-\mathrm{H} / \mathrm{O}-\mathrm{H}$ stretch region.

In the present paper, we provide a brief introduction to another form of vibrational spectroscopy: inelastic neutron scattering (INS) spectroscopy [2]. We then illustrate the complementarity of infrared, Raman and INS spectroscopies with the spectra of L-cysteine. Subsequently, we present new INS spectra of some amino acids and dipeptides. The nature of INS spectra emphasises a 'whole spectrum' approach to vibrational analysis, the strengths and limitations of this method are discussed. We conclude with a forward look.

\footnotetext{
*Corresponding author: Stewart F. Parker, ISIS Facility, STFC Rutherford Appleton Laboratory, Chilton, Didcot, Oxon OX11 0QX, UK. Fax: +44 1235 445720; E-mail: S.F.Parker@rl.ac.uk.
} 


\section{Experimental procedure}

INS spectra were recorded using the spectrometers TOSCA [3] and MARI [4] at ISIS [5]. 2-5 g of the sample were loaded into an aluminium foil sachet, cooled to $20 \mathrm{~K}$ and the spectrum recorded for $\sim 6 \mathrm{~h}$. On MARI, incident energies of 4000, 2000 and $400 \mathrm{~cm}^{-1}$ were used. The spectra recorded on TOSCA are available from the INS database [6,7].

Hartree-Fock calculations were carried out using GAUSSIAN03 [8] with the 6-311 $G(d, p)$ basis set. The output also contains the vibrational frequencies in the harmonic approximation including the atomic displacements for each mode. This enables visualisation of the modes, but more importantly, this is all that is needed in order to generate the INS spectrum using the in-house program ACLIMAX [9] (available from: www.isis.rl.ac.uk/molecularspectroscopy).

All the amino acids and dipeptides were purchased from Aldrich and used as received.

\section{Inelastic neutron scattering spectroscopy}

\subsection{Neutron properties}

The versatility of INS spectroscopy derives from the properties of the neutron, this is an uncharged particle of mass $1.009 \mathrm{amu}$ with a magnetic moment. Since the particle is unchanged, the interaction with matter can be treated as "billiard ball" scattering from the atomic nuclei of the sample and since the nucleus of an atom is only $\sim 1 / 1000$ of the diameter of the atom, this means that neutrons only interact weakly with matter and are consequently highly penetrating. It should be emphasized that neutrons can only properly be described as quantum mechanical entities, thus they exhibit both wave-like and particlelike properties. While the particle-like properties are a convenient picture for the scattering process, the wave-like properties are often exploited for energy selection or analysis. INS spectroscopy spans a wide range in energy, from the equivalent of the microwave region to the $\mathrm{X}$-ray region of the electromagnetic spectrum. Consequently, there are an enormous range of applications [10-12]. This paper will describe the uses of INS for vibrational spectroscopy with energy transfer corresponding to the infrared region, $0-4000 \mathrm{~cm}^{-1}$.

The major difference between vibrational neutron spectroscopy and infrared and Raman spectroscopies is that the neutron has mass, thus an inelastic scattering event results in a significant transfer of both energy $\left(E, \mathrm{~cm}^{-1}\right)$ and momentum $\left(Q, \AA^{-1}\right)$. The energy transfer $\left(E_{\mathrm{T}}\right)$ is given by:

$$
E_{\mathrm{T}}=E_{\mathrm{i}}-E_{\mathrm{f}}
$$

where the subscripts "i" and "f" refer to the incident and final values, respectively. The momentum transfer is given by:

$$
\underline{Q}=\underline{k}_{\mathrm{i}}-\underline{k}_{\mathrm{f}}, \quad \text { where } k \equiv 2 \pi / \lambda,
$$

$k\left(\AA^{-1}\right)$ is the wavevector; $\lambda(\AA)$ is the wavelength of the neutron.

Raman spectroscopy is also an inelastic scattering process, however in this case, the wavelength of both the incident and scattered radiation is several thousand $\AA$, thus the wavevectors are very small so it follows that $Q$ will also be very small. For infrared spectroscopy the incident wavevector is even smaller, since the wavelength is longer and for an absorption process, $k_{\mathrm{f}}$ is zero thus $Q=0$. So both 
infrared and Raman spectroscopy are subject to the selection rule that only transitions at zero wavevector are observable. In contrast, INS spectroscopy is allowed for all wavevectors. Since both $Q$ and $E$ are accessible, it follows that INS spectroscopy is intrinsically a two-dimensional form of spectroscopy.

INS spectroscopy can be either coherent [13], which can be thought of as inelastic diffraction, so gives information on collective motions of the system or incoherent which only involves the correlation between the position of the same nucleus, so there are no interference effects and the motions of a single particle are probed. Whether the scattering is primarily coherent or incoherent depends on the relative size of the coherent and incoherent scattering cross section's $(\sigma)$ of the scattering nuclei. The cross section's are both element and isotope dependent, in particular the incoherent cross section of ${ }^{1} \mathrm{H}$ (hydrogen) is 80.3 barn $\left(1\right.$ barn $\left.=1 \times 10^{-28} \mathrm{~m}^{-2}\right)$, whereas that of most other elements is $<5$ barn. Thus for hydrogenous materials the scattering is dominated by hydrogen. Furthermore, the incoherent cross section of ${ }^{2} \mathrm{H}$ (deuterium) is 5.6 barn so deuteration results in changes in both frequency and intensity. For the remainder of this paper, it will be assumed that the scattering is incoherent.

The INS intensity, $S\left(Q, \omega_{i}\right)$, of the $i$ th mode is proportional to [2]:

$$
S\left(Q, \omega_{i}\right) \propto Q^{2} U_{i}^{2} \exp \left(-Q^{2} U_{\text {Total }}^{2}\right) \sigma
$$

where $U_{i}$ is the amplitude of vibration of the atoms undergoing the particular mode, $\omega_{i}$. The exponential term in Eq. (3) is known as the Debye-Waller factor, $U_{\text {Total }}$ is the mean square displacement of the molecule and its magnitude is in part determined by the thermal motion of the molecule. This can be reduced by cooling the sample and so spectra are typically recorded below $30 \mathrm{~K}$.

\subsection{Instrumentation}

The essential requirement for INS spectroscopy is a source of neutrons. At present there are two routes to producing sufficient neutrons for spectroscopy: via fission of uranium in a reactor or by impacting a high energy proton beam on a metal target (spallation). The world-leading reactor source is the Institut Laue-Langevin at Grenoble, France [14] and the current world-leading spallation source is the ISIS Facility, Chilton, UK [5]. For vibrational spectroscopy, relatively high energy neutrons are required. These are readily available at spallation sources, but only with difficulty at a reactor source. For this reason, most neutron vibrational spectroscopy is carried out at spallation sources.

\subsection{Complementarity of spectra}

Figure 1 shows the infrared, Raman and INS spectra of cysteine. As with all amino acids, this exists as a zwitterion in the solid state. Similarities and differences are apparent between all three types of spectra and emphasise the need to have all three types of spectra for a complete analysis. The similarities arise because the vibrational energy levels are a property of the system, the energy differences between them (the vibrational frequencies) are independent of the method of observation. In contrast, the intensities depend on the technique used to observe the transition. The three forms of vibrational spectroscopy exploit different properties of the molecule. Infrared spectroscopy requires a change in dipole moment, thus is sensitive to the polar motions of the molecule, e.g. the asymmetric carboxylate stretch at $1580 \mathrm{~cm}^{-1}$, while Raman spectroscopy requires a change in polarisability, thus is more sensitive to the non-polar motions of the molecule, e.g. the $\mathrm{C}-\mathrm{C}$ stretch at $639 \mathrm{~cm}^{-1}$ [15]. In contrast, the INS spectrum is dominated by the hydrogenic motions. This arises because the intensity of an INS band is proportional to the 


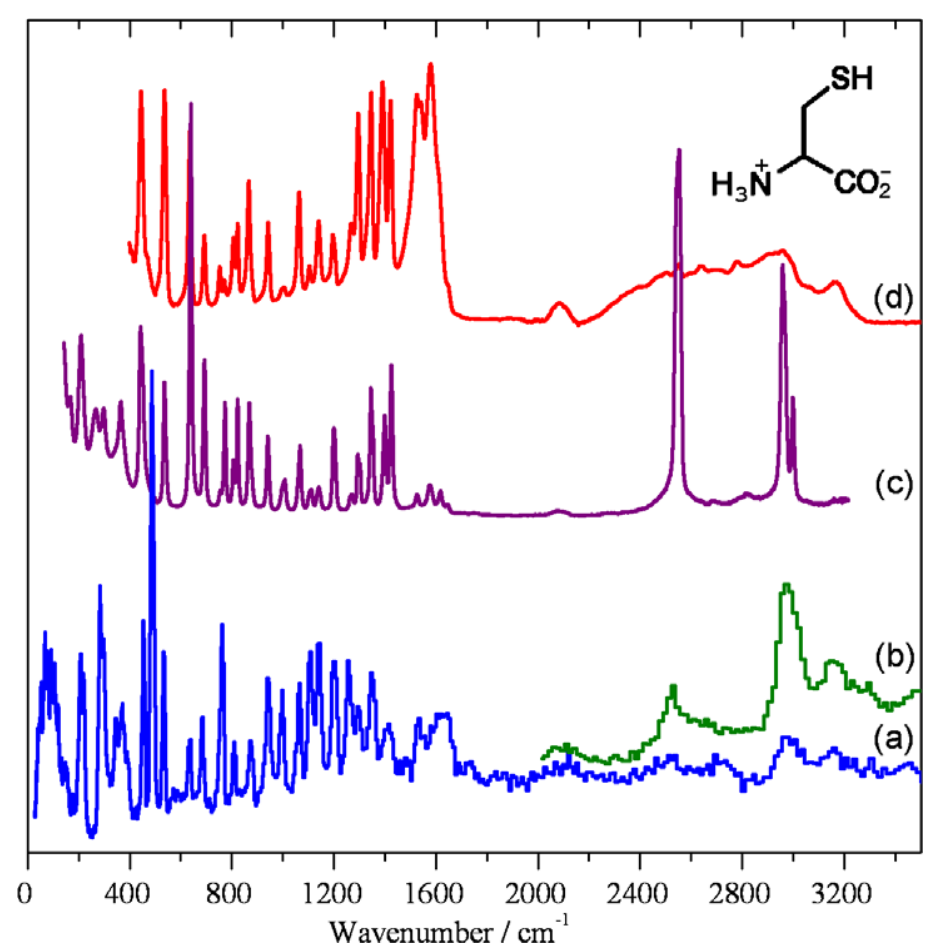

Fig. 1. Vibrational spectra of L-cysteine. (a) INS spectrum recorded on TOSCA, (b) INS spectrum recorded on MARI, (c) Raman spectrum and (d) infrared spectrum.

product of the incoherent scattering cross-section and the amplitude of vibration and is damped by the Debye-Waller factor [2], see Eq. (3). Since the scattering cross-section for hydrogen is at least 20 times larger than for any other type of atom present in the molecule, the INS spectrum emphasises the modes that involve substantial hydrogen motion either directly (e.g. the $\mathrm{CH}_{2}$ rock at $763 \mathrm{~cm}^{-1}$ ) or where the hydrogen is "riding" on another atom (e.g. the $\mathrm{NH}_{3}$ torsion at $487 \mathrm{~cm}^{-1}$ ).

A major difference between INS spectroscopy and infrared and Raman spectroscopies is that there are no selection rules and, in principle, all modes are observable. This arises because the interaction of the neutron is with the nucleus not with the electrons as for the optical methods.

The INS spectra of cysteine shown in Fig. 1(a) and (b) were recorded with two different instruments: TOSCA (a) and MARI (b). As stated earlier, INS spectroscopy is a function of both energy transfer and momentum transfer. The design of TOSCA is such that there is a single momentum transfer associated with each energy transfer, i.e. TOSCA follows a fixed trajectory through $(Q, \omega)$ space. The consequences of this, are that the instrument is very simple to operate and that the resulting spectra are not dissimilar to infrared and Raman spectra, Fig. 1. MARI has a different operating principle and can measure both $Q$ and $\omega$ independently. The price of this is increased complexity and that the incident energy, $E_{\mathrm{i}}$, is fixed, thus it is only possible to measure transitions with an energy less than $E_{\mathrm{i}}$. The $Q$ variation is obtained by varying the scattering angle, so MARI has detectors at $3-130^{\circ}$. Figure 2 shows a MARI plot and its relationship to the TOSCA plot: horizontal lines of intensity correspond to peaks in the TOSCA plot. By examining the variation with $Q$ at constant $\omega$, it is possible to unambiguously determine whether a mode is a fundamental or an overtone [16]. 

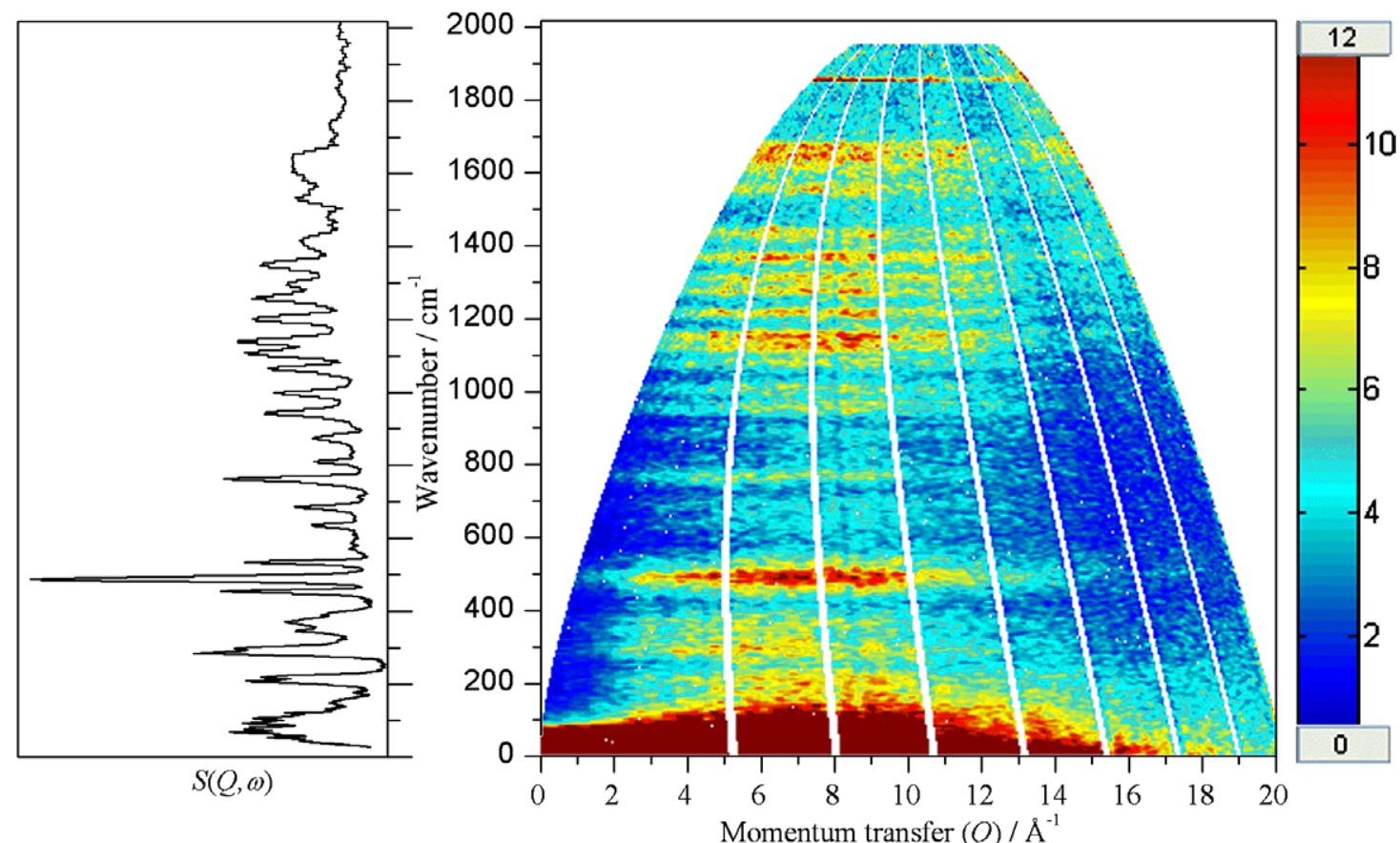

Fig. 2. INS spectra of L-cysteine recorded with MARI (right) and TOSCA (left). Ridges of intensity in the MARI spectrum correspond to peaks in the TOSCA spectrum.

By summing detectors over a range of $Q$, or equivalently scattering angle, the plots in Figs 1(b) and 3(a)-(c) are generated. The TOSCA spectrum is shown in Fig. 3(d) for comparison. The complementarity of the two instruments is readily apparent. MARI can access larger energy transfer than can TOSCA, thus the S-H stretch at $\sim 2500 \mathrm{~cm}^{-1}$ is clearly seen in Fig. 3(a), where it is barely visible in Fig. 3(d). In contrast, the low energy region is much better resolved on TOSCA than on MARI. The resolution behaviour of the two instruments is complementary; on MARI it is a function of $E_{\mathrm{i}}$, thus it degrades from high to low wavenumber and improves as $E_{\mathrm{i}}$ is reduced. On TOSCA, the resolution degrades with increasing wavenumber. For solid systems, where the lines are broadened by a number of factors, the resolution obtainable with TOSCA is comparable to the intrinsic width of the lines. In the high energy region $\left(>2000 \mathrm{~cm}^{-1}\right)$, the resolution obtainable by INS is (and likely always will be) inferior to that of the optical methods. However, the absence of selection rules, means that all the modes appear and to a good approximation, their relative intensity directly reflects their abundance. This is not the case for infrared and Raman spectroscopy and is a feature of INS spectroscopy that is ripe for exploitation.

It is worth noting that INS spectroscopy allows ready access to the low energy or terahertz region $\left(<400 \mathrm{~cm}^{-1}\right)$. On TOSCA, the spectra are routinely obtained down to $30 \mathrm{~cm}^{-1}$, developments in progress will reduce this to less than $10 \mathrm{~cm}^{-1}$. While it is possible to obtain infrared and Raman spectra in this region, it becomes progressively more difficult to do so. Thus infrared spectra are routinely obtained down to $400 \mathrm{~cm}^{-1}$, determined by the $\mathrm{KBr}$ cut-off. Below $400 \mathrm{~cm}^{-1}$ requires a change of beamsplitter and optics. Most Raman instruments employ a notch filter to eliminate the Rayleigh scatter, these typically cut-off in the $100-200 \mathrm{~cm}^{-1}$ range. To obtain spectra below this requires at least a double monochromator with its attendant throughput penalty. 


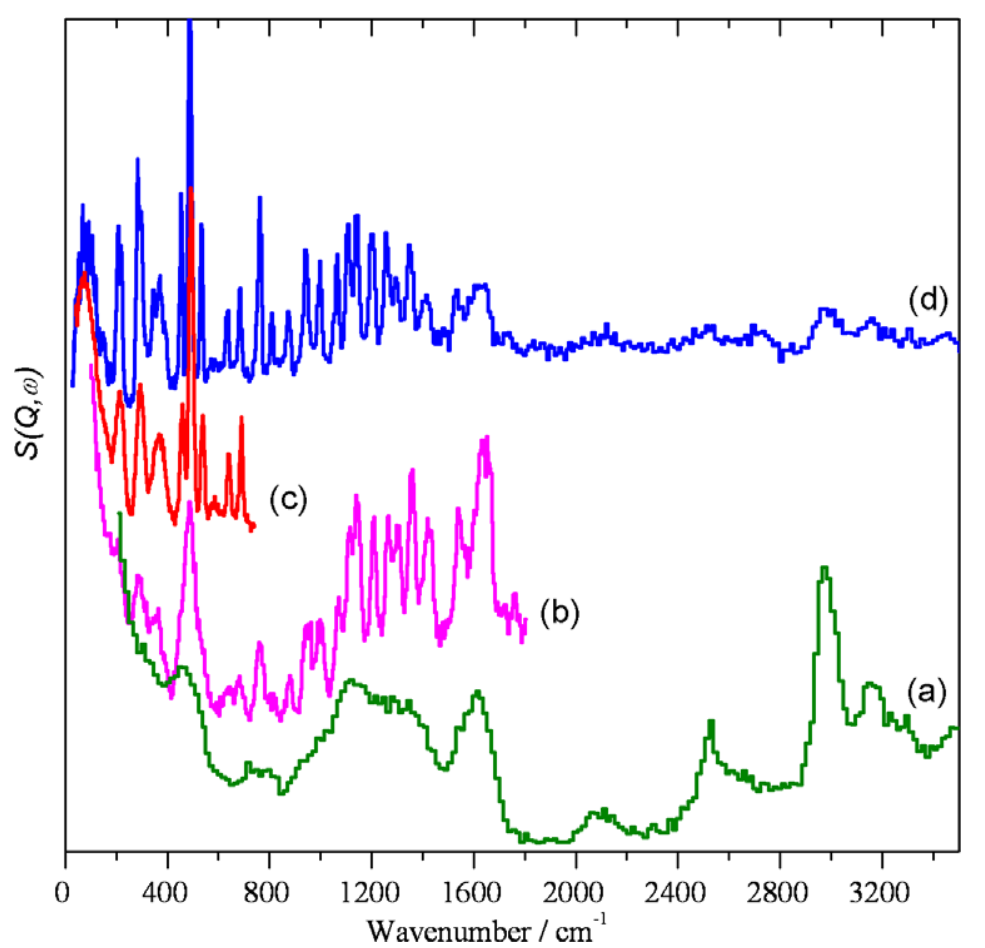

Fig. 3. Comparison of INS spectra of L-cysteine recorded with MARI using an incident energy of: (a) $4000 \mathrm{~cm}^{-1}$, (b) $2000 \mathrm{~cm}^{-1}$, (c) $800 \mathrm{~cm}^{-1}$ and on (d) TOSCA. The resolution on MARI degrades from high to low wavenumber in each spectrum and improves with decreasing incident energy. On TOSCA the resolution degrades with increasing wavenumber.

\subsection{INS spectra of amino acids and dipeptides}

Figure 4 shows the INS spectra of glycine, glutamine and the corresponding dipeptide, Gly-Gln. The strong mode in the $400-500 \mathrm{~cm}^{-1}$ range is the $\mathrm{NH}_{3}$ torsion $[17,18]$. The large number of modes is a consequence of the low symmetry and immediately shows that the modes are highly mixed. It is also clear that the spectra are not simply additive.

A similar picture emerges from the spectra of alanine, phenylalanine and alanine-glycine dipeptide, Gly-Ala, shown in Fig. 5. Two of the few (usually) reliable group frequency modes for INS spectra are present in these spectra. These are the methyl torsion which typically occurs at $200-250 \mathrm{~cm}^{-1}$ when bonded to an aliphatic carbon atom and is often the strongest feature in the spectrum and the out-of-plane phenyl ring deformation near $400 \mathrm{~cm}^{-1}$.

Figure 6 shows the spectra of cysteine, cysteine hydrochloride and methionine. It is apparent that a comparatively small change to cysteine, protonation of the carboxylate group, has resulted in a dramatic change in the spectrum. In the case of methionine, the methyl torsion at $253 \mathrm{~cm}^{-1}$ and the $\mathrm{NH}_{3}$ torsion at $455 \mathrm{~cm}^{-1}$ are readily identified. These are modes that are usually very difficult to detect by infrared and Raman spectroscopy but provide sensitive probes of the local environment.

\subsection{Analysis of INS spectra}

From the previous section, it is clear that the group frequency concept that has played such a major role in optical vibrational spectroscopy is of limited use in the analysis of INS spectra. Partly this is because 


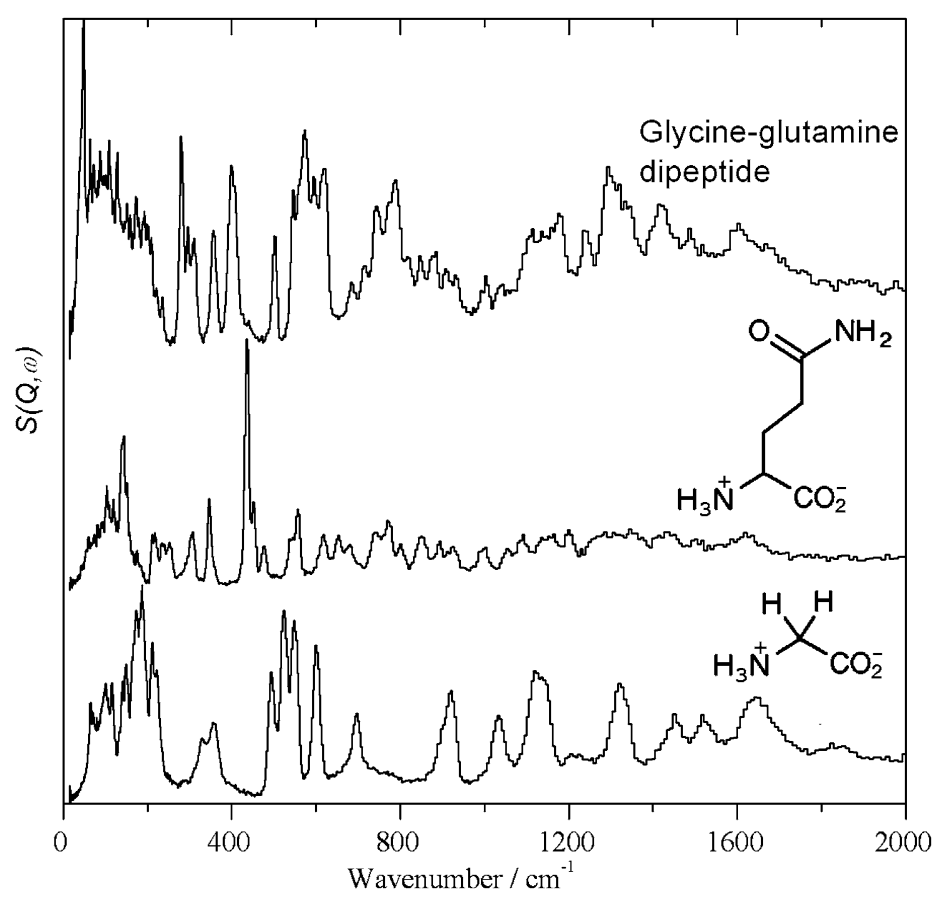

Fig. 4. INS spectra recorded at $20 \mathrm{~K}$ on TOSCA of: glycine (bottom), glutamine (middle) and glycine-glutamine dipeptide (top).

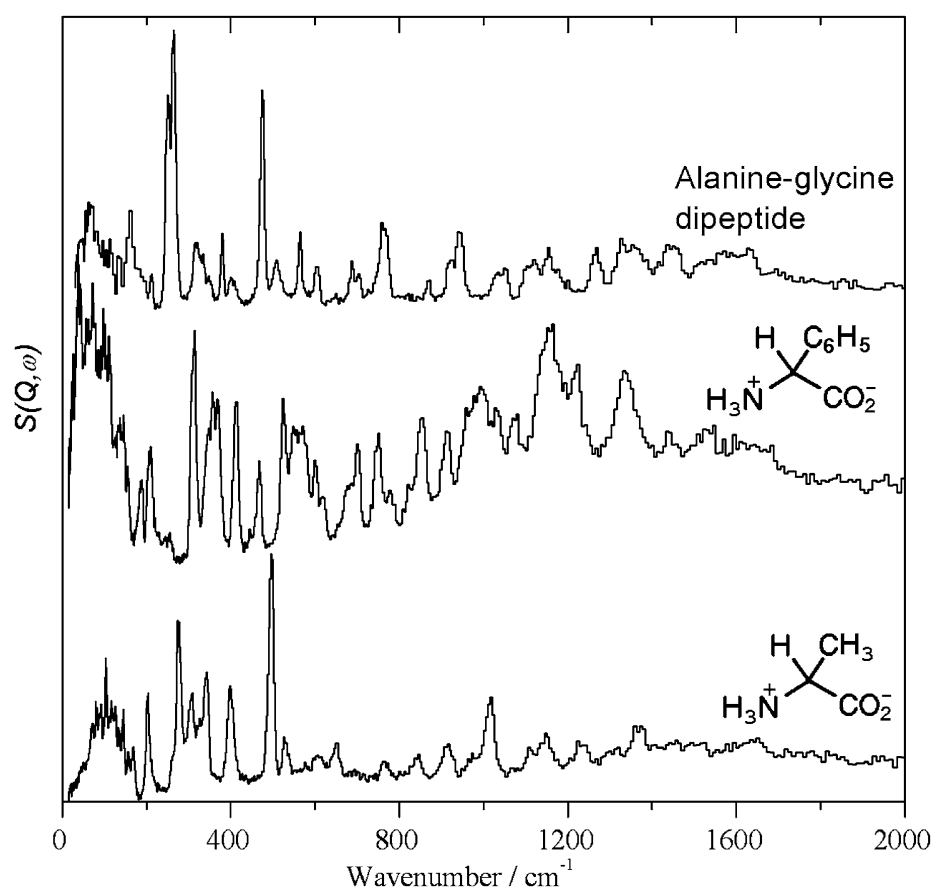

Fig. 5. INS spectra recorded at $20 \mathrm{~K}$ on TOSCA of: alanine (bottom), phenylalanine (middle) and alanine-glycine dipeptide (top). 


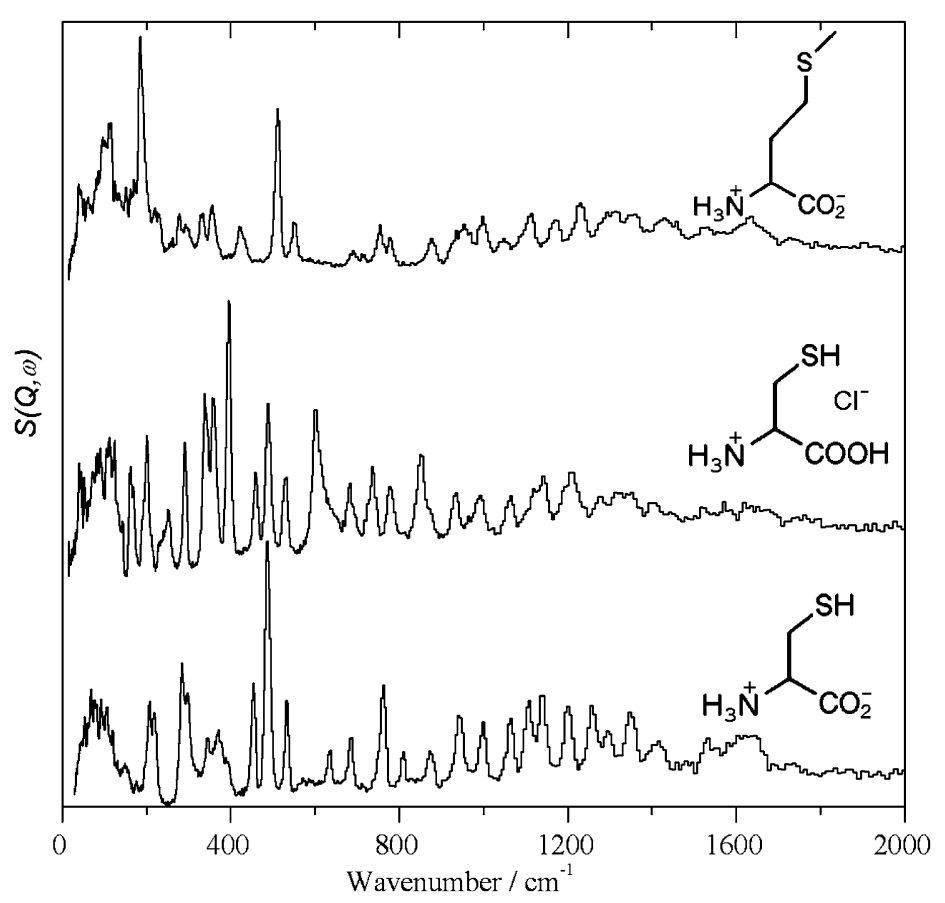

Fig. 6. INS spectra recorded at $20 \mathrm{~K}$ on TOSCA of sulphur-containing amino acids: L-cysteine (bottom), L-cysteine hydrochloride (middle) and methionine (top).

it is only in the last 20 years or so that the resolution of INS spectroscopy has been sufficient to be able to use this approach so there is only a very limited database [7] of spectra to draw on, in comparison to the hundreds of thousand that are available for infrared and Raman spectroscopy. More fundamental is that INS spectroscopy highlights the limitations of the method: particularly in the region below $1500 \mathrm{~cm}^{-1}$ where INS spectroscopy is optimal, there are very few pure modes (except those enforced by symmetry). As noted earlier, this explains the richness of the INS spectra since most modes result in proton motion of some kind and hence INS intensity.

The simplicity of the expression for the intensity of an INS mode, Eq. (3), means that both frequency and intensity can be modelled since only the amplitude of motion is required. This was done initially by methods based on empirical force fields [19] and more recently by ab initio methods. For systems where intermolecular interactions are negligible, isolated (i.e. gas phase) molecule calculations work very well. As an example, the INS spectrum of maleic anhydride [20] calculated by both force field and $a b$ initio methods is shown in Fig. 7. It can be seen that there is little to choose between the two approaches, both give near quantitative agreement with the experimental spectrum.

However, when this approach is applied to alanine it fails disastrously as seen by the comparison shown in Fig. 8. The reason is simple: the isolated molecule approximation is not valid. Alanine is an ionic solid so the intermolecular interactions are particularly strong. Further, in general density functional theory, DFT, gives better agreement between observed and calculated frequencies than does Hartree-Fock theory. The amino acids are unusual in that DFT does not find the zwitterions to be bound species in the gas phase, whereas Hartree-Fock theory does, so this also contributes to the poor agreement. Periodic-DFT calculations of the complete unit cell include the long range Coulombic interactions and so are able to correctly model the system. The lower part of Fig. 8 shows such a very recent cal- 


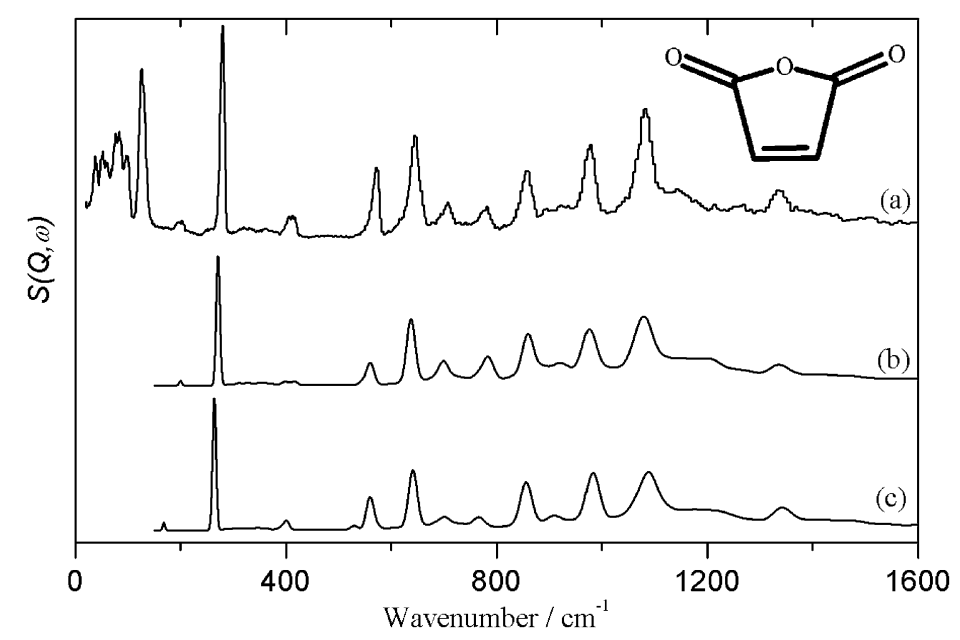

Fig. 7. Comparison of INS spectra of maleic anhydride [20]: (a) experimental, (b) calculated with an empirical force field and (c) isolated molecule ab initio calculation. Reproduced from [20] with permission of the American Chemical Society.

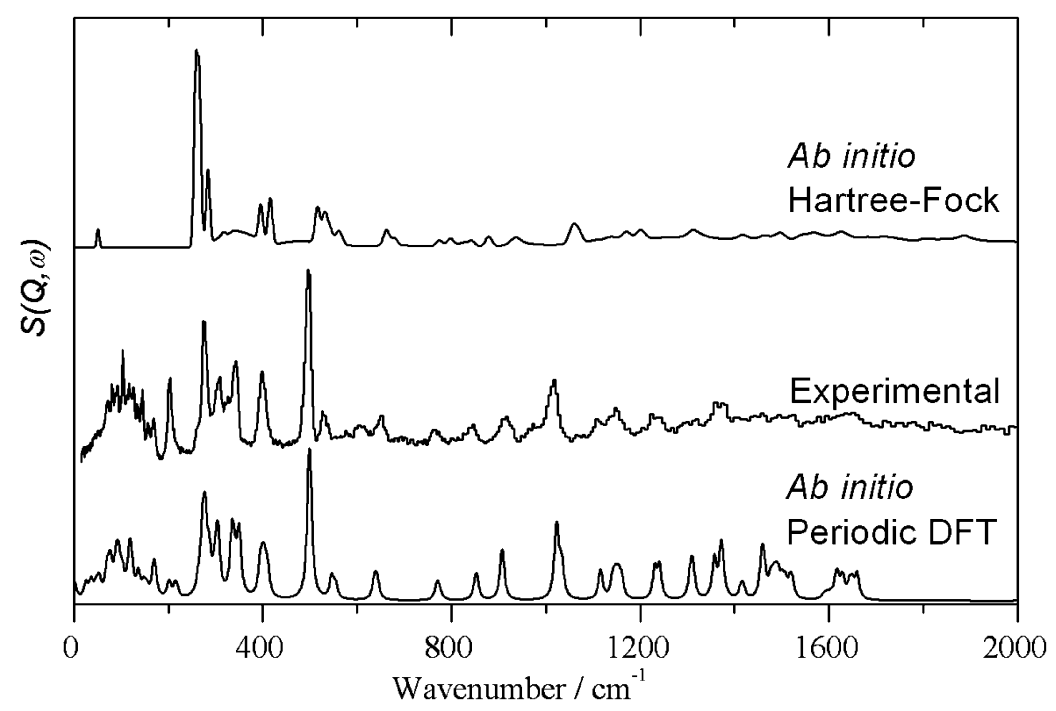

Fig. 8. Comparison of the experimental INS spectrum of L-alanine (centre) with that calculated using the isolated molecule approximation (top) and by periodic-DFT [21] (bottom).

culation [21] for L-alanine. It can be seen that the agreement is remarkable, showing that the DFT has accurately modelled the system, with most of the intensities reproduced to within $20 \%$ and with no scaling of the frequencies.

\section{Conclusions and future directions}

In this paper we aim to have shown how the complementarity of infrared, Raman and INS spectroscopies can be exploited to provide complete vibrational spectra. In particular, the strengths of INS 
spectroscopy are highlighted: the absence of selection rules, that hydrogenic motions are emphasised, the ready access to the low energy regime $\left(<400 \mathrm{~cm}^{-1}\right)$ and the straightforward calculation of intensities.

In principle, the same methods are applicable to larger systems. Thus the INS spectrum of the enzyme, Staphylococcal nuclease, which contains 2395 atoms was modelled [22] with CHARMM, a molecular mechanics programme. As systems become larger, the end groups and the intermolecular interactions become less important, thus it would be realistic to model a protein as an isolated molecule. The largest system modelled [23] to date by ab initio methods is the protein crambin, which has 46 amino acid residues and 1284 atoms. The vibrational spectrum has yet to be calculated but it is only a question of time before this is done, since the limiting factor is computer speed and this type of calculation is readily parallelised. As with small systems, comparison with the INS spectrum will be a stringent test of the calculation.

\section{Acknowledgements}

Prof. Bruce Hudson (Syracuse University) and Prof. Robert Williams are thanked for generously providing the periodic-DFT spectrum of L-alanine. The Rutherford Appleton Laboratory is thanked for access to neutron beam facilities.

\section{References}

[1] R. Schweitzer-Stenner, Vib. Spec. 42 (2006), 98-117.

[2] P.C.H. Mitchell, S.F. Parker, A.J. Ramirez-Cuesta and J. Tomkinson, Vibrational Spectroscopy with Neutrons, with Applications in Chemistry, Biology, Materials Science and Catalysis, World Scientific, Singapore, 2005.

[3] Z.A. Bowden, M. Celli, F. Cilloco, D. Colognesi, R.J. Newport, S.F. Parker, F.P. Ricci, V. Rossi-Albertini, F. Sacchetti, J. Tomkinson and M. Zoppi, Physica B 276-278 (2000), 98-99.

[4] M. Arai, A.D. Taylor, S.M. Bennington and Z.A. Bowden, in: Recent Developments in the Physics of Fluids, W.S. Howells and A.K. Soper, eds, Adam Hilger, Bristol, 1992, pp. F321-F328.

[5] http://www.isis.rl.ac.uk.

[6] S.F. Parker and D.J. Champion, Internet J. Vib. Spec. 3 (1999), http://www.ijvs.com/volume3/edition3/section1.htm\#Ed.

[7] www.isis.rl.ac.uk/insdatabase.

[8] M.J. Frisch, G.W. Trucks, H.B. Schlegel, G.E. Scuseria, M.A. Robb, J.R. Cheeseman, J.A. Montgomery Jr., T. Vreven, K.N. Kudin, J.C. Burant, J.M. Millam, S.S. Iyengar, J. Tomasi, V. Barone, B. Mennucci, M. Cossi, G. Scalmani, N. Rega, G.A. Petersson, H. Nakatsuji, M. Hada, M. Ehara, K. Toyota, R. Fukuda, J. Hasegawa, M. Ishida, T. Nakajima, Y. Honda, O. Kitao, H. Nakai, M. Klene, X. Li, J.E. Knox, H.P. Hratchian, J.B. Cross, V. Bakken, C. Adamo, J. Jaramillo, R. Gomperts, R.E. Stratmann, O. Yazyev, A.J. Austin, R. Cammi, C. Pomelli, J. Ochterski, P.Y. Ayala, K. Morokuma, G.A. Voth, P. Salvador, J.J. Dannenberg, V.G. Zakrzewski, S. Dapprich, A.D. Daniels, M.C. Strain, O. Farkas, D.K. Malick, A.D. Rabuck, K. Raghavachari, J.B. Foresman, J.V. Ortiz, Q. Cui, A.G. Baboul, S. Clifford, J. Cioslowski, B.B. Stefanov, G. Liu, A. Liashenko, P. Piskorz, I. Komaromi, R.L. Martin, D.J. Fox, T. Keith, M.A. Al-Laham, C.Y. Peng, A. Nanayakkara, M. Challacombe, P.M.W. Gill, B.G. Johnson, W. Chen, M.W. Wong and C. Gonzalez, GAUSSIAN 03 (Revision B.05), Gaussian Inc., Pittsburgh, PA, 2003.

[9] A.J. Ramirez-Cuesta, Comp. Phys. Comm. 157 (2004), 226-238.

[10] M. Bée, Quasielastic Neutron Scattering, Adam Hilger, Bristol, 1988.

[11] R.J. Newport, B.D. Rainford and R. Cywinski (eds), Neutron Scattering at a Pulsed Source, Adam Hilger, Bristol, 1988.

[12] J.S. Higgins and H.C. Benoît, Polymers and Neutron Scattering, Oxford University Press, Oxford, 1994.

[13] G. Shirane, S.M. Shapiro and J.M. Tranquada, Neutron Scattering with a Triple-Axis Spectrometer, Cambridge University Press, Cambridge, 2002.

[14] http://www.ill.fr.

[15] A. Pawlukojć, J. Leciejewicz, A.J. Ramirez-Cuesta and J. Nowicka-Scheibe, Spec. Acta A 61 (2005), 2474-2481.

[16] S.F. Parker, S.M. Bennington, A.J. Ramirez-Cuesta, G. Auffermann, W. Bronger, H. Herman, K.P.J. Williams and T. Smith, J. Am. Chem. Soc. 125 (2003), 11656-11661. 
[17] D. Lin-Vien, N.B. Colthup, W.G. Fateley and J.G. Grasselli, The Handbook of Infrared and Raman Characteristic Frequencies of Organic Molecules, Academic Press, San Diego, 1991.

[18] A.M. Amado, J.C. Otero, M.P.M. Marques and L.A.E. Batista de Carvalho, Chem. Phys. Chem. 5 (2004), 1837-1847.

[19] G.J. Kearley, Nucl. Inst. Methods Phys. Res. A 354 (1995), 53-58.

[20] S.F. Parker, C.C. Wilson, J. Tomkinson, D.A. Keen, K. Shankland, A.J. Ramirez-Cuesta, P.C.H. Mitchell, A.J. Florence and N. Shankland, J. Phys. Chem. A 105 (2001), 3064-3070.

[21] R.W. Williams, S. Schlücker and B.S. Hudson, Chem. Phys. 343 (2008), 1-18.

[22] A.V. Goupil-Lamy, J.C. Smith, J. Yunoki, F. Tokunaga, S.F. Parker and M. Kataoka, J. Am. Chem. Soc. 119 (1997), 9268-9273.

[23] http://www.hpcx.ac.uk/highlights/Bush_CapCom_Autumn03_No2.pdf. 


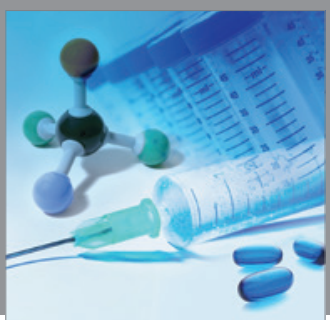

International Journal of

Medicinal Chemistry

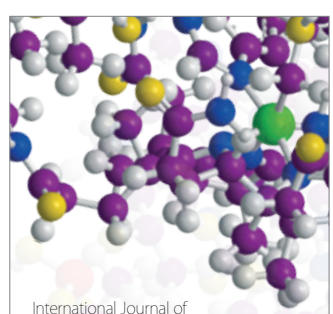

Carbohydrate Chemistry

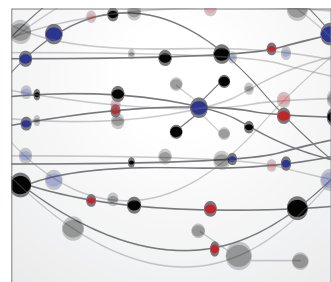

The Scientific World Journal
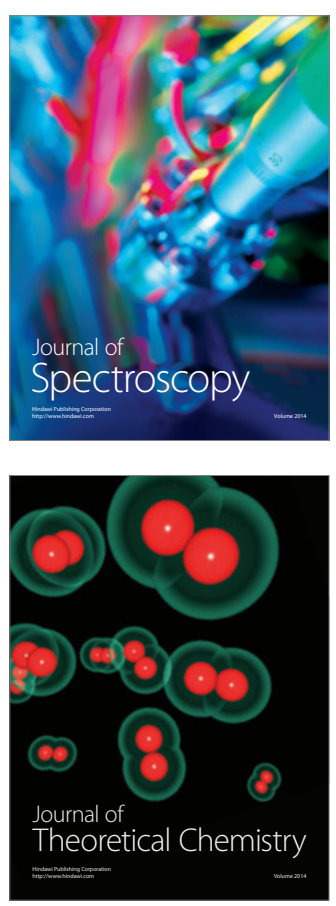
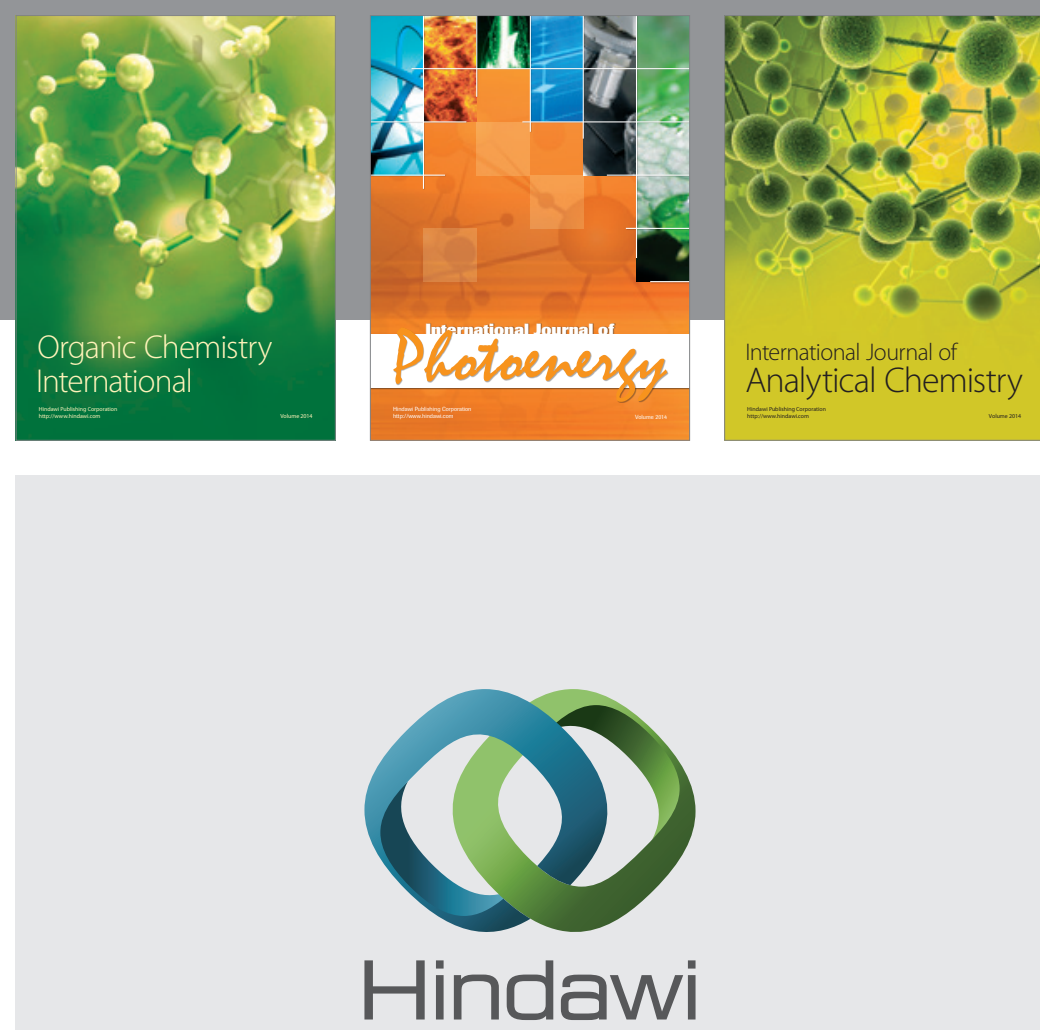

Submit your manuscripts at

http://www.hindawi.com
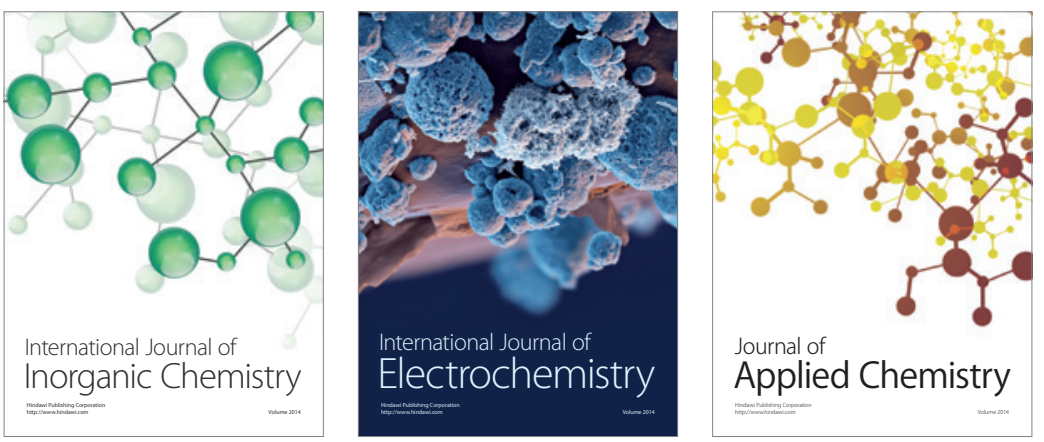

Journal of

Applied Chemistry
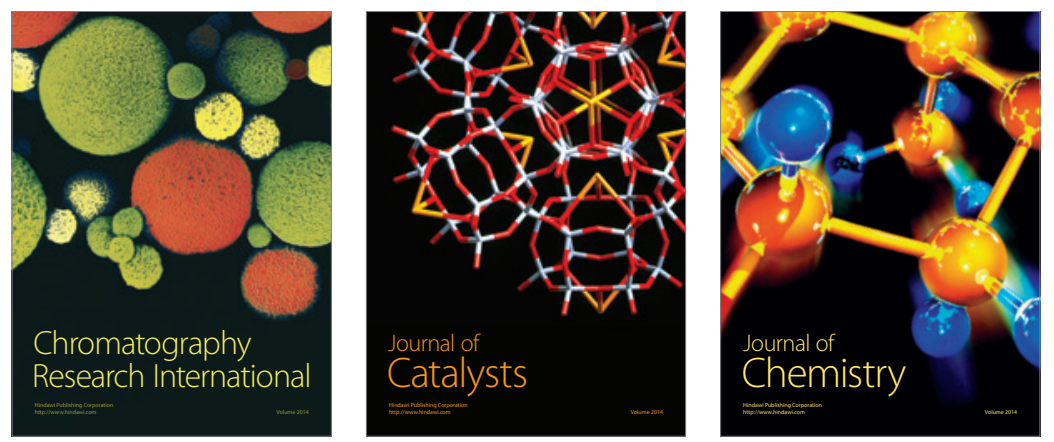
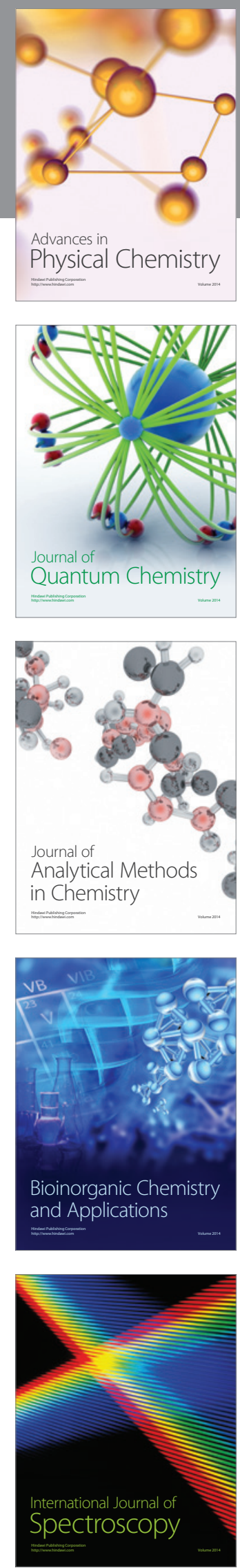